\title{
Evaluation of radial Extracorporeal Shock Wave Therapy (rESWT) as a treatment option in chronic post- stroke upper limb management
}

\author{
Senarath MKID \\ Department of Physiotherapy, Faculty of Allied Health Sciences, University of Peradeniya, Sri Lanka, 20000 \\ MSc in Applied Epidemiology (PDN), B.Sc in Physiotherapy (PDN) \\ DOI: 10.29322/IJSRP.11.05.2021.p11334 \\ http://dx.doi.org/10.29322/IJSRP.11.05.2021.p11334
}

\begin{abstract}
Background: Stroke leaves almost fifty percent (50\%) of its survivors disabled with respect to arm-hand performance, and after six months, $25-53 \%$ of people are dependent at least one ADL task. Shock wave therapy used in treating post-stroke upper limb for many years; therefore this current review discusses the literature on the effects of shock wave therapy on chronic poststroke upper limb management.
\end{abstract}

Materials and Methods: A literature survey was conducted through MEDLINE PubMed, PEDro, Science Direct and Google Scholar. 'Chronic stroke, spasticity, upper limb and shock wave therapy' were used as the key words independently and/ or combination and included articles only written in English.

Results: Two application sites for rESWT treatment were identified as muscle belly and musculotendinous junction. Due to its elcetrophysiogical and thermal effects, rESWT treatment should be considered as a possible anti-spastic effect to regulate vasculature. On its long lasting effect with a single treatment session, there were conflicting results as 12 weeks (2005), 5 weeks (2015) and 8 weeks (2016). With two sessions of rESWT, improvements were maintained up to 6 months and with three sessions, persistent effects were observed up to 16 weeks. All the studies used Modified Ashwath scale (MAS) as the primary outcome measure for spasticity.

Conclusion: All the studies conclude that the rESWT is an effective treatment method for treating post-stroke upper limb. There were conflicts of the long-lasting effects with single session, two and three sessions, therefore further investigations are needed to clarify those with larger sample size.

Index Terms- chronic stroke, upper limb, spasticity, rESWT

\section{INTRODUCTION}

$\mathrm{S}$ troke leaves almost fifty percent $(50 \%)$ of its survivors disabled with respect to arm-hand performance, often for the rest of their lives ${ }^{1,2}$. From the disabled stroke survivors, twenty to eighty percent of patients show incomplete recovery depending on the initial impairment ${ }^{3,4}$. Upper-limb dysfunction in stroke is characterized by paresis, loss of manual deftness, and movement abnormalities that may influence significantly on the performance of activities of daily livings (ADLs) ${ }^{5}$. Six months after the stroke episode, a considerable number (25-53\%) of people who survive are dependent at least one ADL task, which frequently comprises the use of unilateral or bilateral upper limb movement ${ }^{6}$.

Recently, some of the studies reported that shock wave therapy (SWT) or radial extracorporeal shock wave therapy (rESWT) is a safe, noninvasive, substitute treatment for poststroke hemiplegia management ${ }^{7}$. Radial extracorporeal shock wave therapy (rESWT) can be defined as "a sequence of acoustic pulses characterized by high peak pressure (100 MPa), fast pressure rise $(<10 \mathrm{~ns})$, short duration $(10 \mu \mathrm{s})$, and an energy density ranging from 0.003 to $0.890 \mathrm{~mJ} / \mathrm{mm}$ ". Clinical experiments and other different studies proved the efficacy of ESWT in the treatment of musculoskeletal disorders such as chronic tendinopathies, calcific tendinitis of the shoulder, lateral epicondylitis, and plantar fasciitis, etc. The side effects of ESWT including aching, tingling, redness, or bruising are relatively rare and transitory ${ }^{8}$.

There are numerous studies conducted on different effects on shock wave therapy; therefore this current review discusses the literature on the effects of shock wave therapy on chronic poststroke upper limb management.

\section{MATERIALS AND METHODS}

A literature survey was conducted by the author through the electronic data bases such as; MEDLINE PubMed, PEDro, Science Direct and Google Scholar. 'Chronic stroke, spasticity, upper limb and shock wave therapy' were used as the key words independently and/ or combination. All the selected articles were published in English. Studies investigating the effects of shock wave therapy (SWT) on chronic post-stroke upper limb management were included in this review. All together eight (8) randomized controlled trials (RCTs) were included which were based on different effects on shock wave therapy on upper limb.

\section{RESUlTS AND DISCUSSION}

\section{Site of Application}

There were two (02) sites identified with shock wave application; muscle belly/ group and musculotendinous/ myotendinous junction. A South Korean study was found which conducted to compare the effects of extracorporeal shock wave therapy (ESWT) according to the study site in 2010. Thirty two 
(32) patients with elbow flexor spasticity after stroke was taken as the study participants; ESWT was applied one session/week, total three sessions at biceps muscle belly to 12 patients and at biceps musculotendinosus junction to 11 patients and 9 were included in control group . Patients were evaluated using modified Ashworth scale (MAS), modified Tardieu scale (MTS), Korean-modified Barthel index (K-MBI) at baseline, immediately, 1 week and 4 weeks after ESWT. It has been concluded that the treatment effect was greater in musculotendinous junction group than muscle belly group in MTS and MAS immediately after ESWT ${ }^{9}$. Another South Korean study was done in 2017 with 80 patients (muscle belly, myotendinous and control groups) with stroke-induced spasticity on the elbow flexor; a total of three sessions, one session per week $\left(0.068-0.093 \mathrm{~mJ} / \mathrm{mm}^{2}, 1,500\right.$ shots $)$ was applied at the two application sites and assessed by MAS and MTS at the baseline and at 1 week after each session. In this study it was identified as no significant difference between the muscle belly and myotendinous groups ${ }^{10}$.

\section{Electrophysiological and thermal effects of rESWT}

A study conducted in Poland, 2016 has assigned 30 patients for placebo rESWT and 30 patients for active rESWT treatment; surface electromyography (sEMG) was performed for the resting bioelectrical activity registration of the flexor carpi radialis (FCR) and flexor carpi ulnaris (FCU) muscles; and infrared thermal imaging (IRT) was used to assess the temperature distributions of the carpal flexor muscles (CFM). All assessments were performed at baseline (t0), immediately after rESWT (t1) as well as 1 hour and 24 hours following at the end of the application (t2 and $\mathrm{t} 3$ ). Patients treated with active rESWT have shown a significant decrease in sEMG activity was observed in the FCR and FCU muscles at $\mathrm{t} 1, \mathrm{t} 2$ and $\mathrm{t} 3(\mathrm{p}<0.05)$; significant increases in the mean and maximum values of the IRT detection was observed using inter-group comparisons in $\mathrm{t} 2$ and $\mathrm{t} 3(\mathrm{p}<0.05)$. Therefore, this study concluded that the rESWT treatments should be considered as a potential anti-spastic effect to regulate vasculature $^{11}$.

\section{Long lasting effects from application of a single session}

A study conducted in Italy in 2005, investigated the effect and duration of ESWT on spasticity of the hand and wrist. Twenty stroke patients were enrolled with severe hypertonia in upper limbs. First, a placebo stimulation was performed and after 1 week of it, active stimulation was done in each patient. Evaluation was performed using the National Institutes of Health (NIH), MAS and video monitoring with a digital goniometer before and immediately after placebo or active stimulation. Motor nerve conduction velocity from abductor digiti minimi was recorded. Patients were examined at 1, 4, and 12 weeks after active treatment. Patients showed greater improvement in flexor tone of wrist and fingers after active ESWT compared with placebo stimulation. At the 1- and 4-week follow-up visits, a significant decrease of passive muscle tone was noted on muscles in all patients receiving active treatment. At 12 weeks after therapy, 10 of the 20 patients showed persistent reduction in muscle tone. Therefore, it was concluded as ESWT reduces hypertonia of the wrist and finger muscles for 12 weeks after treatment ${ }^{12}$.
An Iranian study on post stroke wrist flexor spasticity was conducted on 2015 with 15 patients ( 12 male and 3 female with a mean age of 54 years) to evaluate the effects of single session. Patients received 1 sham ESWT followed by 1 active ESWT 1 week later. Modified Modified Ashworth Scale (MMAS), the Hmax/Mmax ratio, and the Brunnstrom motor recovery stage were taken as the outcome measures. No significant effects with sham ESWT. After active ESWT, the MMAS scores of spasticity and the Hmax/Mmax ratio improved. The improvements were maintained up to five (5) weeks with active ESWT. The study was concluded that in adult patients after stroke, a single session of active ESWT resulted in significant improvement in the wrist flexor spasticity and alpha motor neuron excitability ${ }^{13}$.

A Poland study was conducted in 2016, on wrist and fingers flexors spasticity in stroke with 20 patients. Modified Ashworth Scale (MAS) was used to assess the spasticity level of the radio carpal (RC) and finger (FF) joints. Measurements were taken at baseline $(t 0)$, immediately after $\operatorname{rESWT}(t 1)$, and $1(t 2)$ and $24(t 3)$ hours following rESWT. Significant reduction in MAS was observed for the $\mathrm{RC}$ joint in $t 1$, as well as for the FF joints in $t 1, t 2$, and $t 3$. The conclusion of the study was, a single session of rESWT could be an effective alternative treatment for limb spasticity reduction, but the long term follow-up was not conducted to identify the maintenance of improvement with one application of $\mathrm{rESWT}^{14}$.

A Taiwan study in 2016 assessed the long lasting effects of single rESWT treatment with 20 patients. MAS and FuglMeyer Assessment of hand function and wrist control was taken as the assessment tools. Evaluations were performed before the rESWT treatment and immediately 1, 4, 8, 12, and 16 weeks after the session. Significant reduction in spasticity of hand and wrist lasted at least 8 weeks. The study concluded that even with one session there are long lasting effects with rESWT of the upper limb in chronic stroke patients ${ }^{15}$.

\section{Long lasting effects from application of several sessions} Two Sessions

Another Italian study in 2013 was conducted to evaluate the long-term effects of ESWT on muscle tone and motricity in upper limb in stroke patients. Selected 12 patients were treated with two sessions of ESWT. Participants were assessed at baseline, after the treatment, and at 3 and 6 months. MAS was used to assess the spasticity and motor recovery was assessed by motricity, passive range of motion (PROM) and pain sub-scores of upper extremity part of the Fugl-Meyer scale of shoulder adductors, elbow, wrist and finger flexors. Visual Analogue Scale (VAS) was used to assess the degree of perceived benefit from treatment protocol. MAS showed a significant reduction of spasticity and Fugl-Meyer scores improved immediately after treatment. Persistent effects were observed at 3 and 6 months for MAS, and for motricity and PROM sub-scores of the Fugl-Meyer scale. Clinical improvement was not correlated to the patients' perceived benefit. It was concluded two sessions of ESWT seem to have long-term effects in reducing muscle tone and enhancing motor impairment from this study ${ }^{16}$.

Three sessions 
A prospective, randomized, single blind, placebocontrolled study was conducted in Taiwan 2016 with 40 patients in two groups; one groups received one session of rESWT per week for three weeks consecutively. Other groups receives sham rESWT per week for three consecutive weeks. MAS of hand and wrist was taken as the primary outcome and Fugl-Meyer Assessment of hand function and wrist control was taken as the secondary outcome. Assessments were performed before the first rESWT treatment and immediately 1, 4, 8, 12, and 16 weeks after the last session of rESWT. Experimental group has shown a significant reduction in spasticity of hand and wrist lasted at least 16 weeks compared with the control group. Three sessions had a longer lasting effect than one session and the reduction in spasticity after 3 sessions of rESWT may be beneficial for hand function and wrist control and the effect was maintained for 16 and 12 weeks, respectively. This study concluded that the rESWT may be valued in decreasing spasticity of the hand and wrist with associated improvement of wrist control and hand function in chronic stroke patients ${ }^{15}$.

\section{Outcome measures}

All the evaluated RCTs has taken Modified Ashwath scale (MAS) as their primary outcome measure to assess spasticity. There were two South Korean studies, both were used modified Tardieu scale (MTS) and Korean-modified Barthel index (K-MBI) as their outcome measures along with MAS ${ }^{9,10}$. Two studies has taken Fugl-Meyer Assessment of hand function and wrist control as their secondary outcome measure ${ }^{15,16}$ while another study was utilized Hmax/Mmax ratio and the Brunnstrom motor recovery stage $^{13}$. National Institutes of Health (NIH), video monitoring with a digital goniometer ${ }^{12}$, motricity, passive range of motion (PROM), VAS ${ }^{16}$, Surface electromyography (sEMG), infrared thermal imaging (IRT) ${ }^{11}$ were applied as other outcome measures.

\section{DISCUSSION}

For reduction in post-stroke upper limb spasticity, rESWT is a promising treatment option which is safe and free of adverse side effects. The mechanism behind the action of rESWT of spastic muscle is still not clear ${ }^{17}$ but one study was mentioned that there were a potential anti-spastic effect to regulate vasculature with $\mathrm{rESWT}^{11}$.

There were two studies conducted on the site of application of rESWT, the study conducted in 2010 with 32 patients, was identified musculotendinous junction group improved more compared with muscle belly group. The study done in 2017 suggest that there were no such effects which was conducted with comparably large sample size as 80 patients. As a recent study with fairly large sample size, it can be suggested as there are no difference either musculotendinous junction or muscle belly as the application site. In this literature review, the long lasting effects from a single application was identified as five weeks, eight weeks and twelve weeks in three different studies. Less than 20 patients were included in each study. Long lasting effects may be vary due to the difference of application site, applied muscle, dose, duration etc. ${ }^{2,9}$
Another study was found with two sessions of rESWT application, it has shown that there were a significant reduction of spasticity with MAS and Fugl-Meyer scores just after the treatment. Follow-up assessments were conducted at $3^{\text {rd }}$ and $6^{\text {th }}$ month after the second session and has observed persistent effects ${ }^{16}$. With three sessions of the treatment, it was observed that spasticity reduction is maintained up to 16 weeks ${ }^{15}$. Therefore, it is uncertain to conclude the long lasting effects of rESWT it may depend on the number of applications. In almost all the studies, rESWT was applied once a week. Regardless of the number of treatment sessions, a systematic review (2019) and a meta-analysis (2020) was conducted on its long lasting effects. It has been concluded that rESWT effectively reduced spasticity levels measured with $\mathrm{MAS}^{18,19}$ while reducing pain, enhancing ROM and motor function in stroke patients ${ }^{19}$ and effect maintained for 12 weeks ${ }^{18}$. The systematic review further concluded that the number of shocks or site of application had no significant influence on the therapeutic effect of ESWT in spasticity reduction $^{18}$.

\section{CONCLUSION}

rESWT is an effective treatment method of treating poststroke upper limb spasticity. Though there are two application sites, the treatments effects were not depend on that. Regardless of the number of sessions, usually rESWT is applying once a week. As it is conflicting with the long lasting effects with one, two or three sessions of application, RCT with large number of study participants will be suggested. There are no standard parameters of rESWT was established regarding intensity, frequency, number of applications, and applied muscle. Further clinical trials are needed to conduct on that to establish the parameters.

There is no conflict of interest.

\section{REFERENCES}

[1] Broeks JG, Lankhorst GJ, Rumping K, Prevo AJ. The longterm outcome of arm function after stroke: results of a followup study. Disabil Rehabil. 1999;21:357-364

[2] Shelton FN, Reding MJ. Effect of lesion location on upper limb motor recovery after stroke. Stroke. 2001;32:107-112.

[3] Nakayama H, Jorgenson HS, Raaschou HO, Olsen TS. Recovery of upper extremity function in stroke patients: the Copenhagen stroke study. Arch Phys Med Rehabil. 1994;75:394-8.

[4] Parker VM, Wade DT, Langton Hewer R. Loss of arm function after stroke: measurement, frequency, and recovery. Int Rehabil Med. 1986;8:69-73.

[5] Sveen U, Bautz-Holter E, Sødring KM, Wyller TB, Laake K. Association between impairments, self-care ability and social activities 1 year after stroke. Disabil Rehabil. 1999;21:372-7.

[6] Blennerhassett J, Dite W. Additional task-related practice improves mobility and upper limb function early after stroke: a randomised controlled trial. Aust J Physiother. 2004; 50(4):219-224

[7] Li TY, Chang CY, Chou YC, Chen LC, Chu HY, Chiang SL et al. Effect of radial shock wave therapy on spasticity of the upper limb in patients with chronic stroke: A prospective, randomized, single blind, control trial Medicine (Baltimore). 2016 May 6; 95(18): e3544. Pubmed PMID: PMC 4863782 .

[8] Gerdesmeyer L, Wagenpfeil S, Haake M, et al. Extracorporeal shock wave therapy for the treatment of chronic calcifying tendonitis of the rotator cuff: a randomized controlled trial. JAMA. 2003; 290:2573-2580 
[9] Bae H, Lee JM, Lee KH. The effects of Extracorporeal Shock Wave Therapy on Spasticity in Chronic Stroke Patients. J Korean Rehab Med. 2010;34:663669.

[10] Yoon SH, Shin MK, Choi EJ, Kang HJ. Effective Site for the Application of Extracorporeal Shock-Wave Therapy on Spasticity in Chronic Stroke: Muscle Belly or Myotendinous Junction. Ann Rehabil Med. 2017;41(4):547555. doi:10.5535/arm.2017.41.4.547

[11] Dymarek R, Taradaj J, Rosińczuk J. The Effect of Radial Extracorporeal Shock Wave Stimulation on Upper Limb Spasticity in Chronic Stroke Patients: A Single-Blind, Randomized, Placebo-Controlled Study. $\begin{array}{llll}\text { Ultrasound } & \text { Med } & \text { Biol. } & \text { 2016;42(8):1862-1875. }\end{array}$ doi:10.1016/j.ultrasmedbio.2016.03.006

[12] Manganotti P, Amelio E. Long-term effect of shock wave therapy on upper limb hypertonia in patients affected by stroke. Stroke. 2005;36(9):1967-1971. doi:10.1161/01.STR.0000177880.06663.5c

[13] Daliri SS, Forogh B, Emami Razavi SZ, Ahadi T, Madjlesi F, Ansari NN. A single blind, clinical trial to investigate the effects of a single session extracorporeal shock wave therapy on wrist flexor spasticity after stroke. NeuroRehabilitation. 2015;36(1):67-72. doi:10.3233/NRE-141193

[14] Dymarek R, Taradaj J, Rosińczuk J. Extracorporeal Shock Wave Stimulation as Alternative Treatment Modality for Wrist and Fingers Spasticity in Poststroke Patients: A Prospective, Open-Label, Preliminary Clinical Trial. Evid Based Complement Alternat Med. 2016;2016:4648101. doi:10.1155/2016/4648101

[15] Li TY, Chang CY, Chou YC, et al. Effect of Radial Shock Wave Therapy on Spasticity of the Upper Limb in Patients With Chronic Stroke: A Prospective, Randomized, Single Blind, Controlled Trial. Medicine (Baltimore). 2016;95(18):e3544. doi:10.1097/MD.0000000000003544
[16] Troncati F, Paci M, Myftari T, Lombardi B. Extracorporeal Shock Wave Therapy reduces upper limb spasticity and improves motricity in patients with chronic hemiplegia: a case series. NeuroRehabilitation. 2013;33(3):399405. doi:10.3233/NRE-130970

[17] Opara J, Taradaj J, Walewicz K, Rosińczuk J, Dymarek R. The Current State of Knowledge on the Clinical and Methodological Aspects of Extracorporeal Shock Waves Therapy in the Management of Post-Stroke SpasticityOverview of 20 Years of Experiences. J Clin Med. 2021;10(2):261. Published 2021 Jan 12. doi: $10.3390 / \mathrm{jcm} 10020261$

[18] Ho Oh J, Park HD, Han SH, Shim GY, Choi KY. Duration of treatment Effect of Extracorporeal Shock Wave on Spasticity and Subgroup-Analysis According to Number of Shocks and Application Site: A Meta-Analysis. Ann Rehabil Med. 2019;43(2):163-177. doi:10.5535/arm.2019.43.2.163

[19] Jia G, Ma J, Wang S, et al. Long-term Effects of Extracorporeal Shock Wave Therapy on Poststroke Spasticity: A Meta-analysis of Randomized Controlled Trials. J Stroke Cerebrovasc Dis. 2020;29(3):104591. doi:10.1016/j.jstrokecerebrovasdis.2019.104591

\section{AUTHORS}

First Author - Senarath MKID, Lecturer, Department of Physiotherapy, Faculty of Allied Health Sciences, University of Peradeniya, Sri Lanka, 20000, MSc in Applied Epidemiology (PDN), B.Sc in Physiotherapy (PDN), dilharisenarath@pdn.ac.lk/ dilsenarath7@gmil.com 